\title{
Performance Analysis of HSTCP for Optimizing Data Transfer rate in Mobile Ad-hoc Networks
}

\author{
Gururaj H.L. \\ Research Scholar \\ Department of CS\&E \\ Malnad College of Engineering, Hassan, India
}

\author{
Ramesh B. \\ Professor \\ Department of CS\&E \\ Malnad College of Engineering, Hassan, India
}

\begin{abstract}
In the era of communication congestion is the majorproblemwhich degrades the performance. In Ad hoc networks due to infrastructure less character there is no guaranteed bandwidth and node mobility. The traditional TCP variants were good for wired environment, whereas in Ad-hoc networks there are lots of other factors to be considered. The congestion window is one of the factors that determine the number of bytes that can be outstanding at any time. The congestion window indicates the maximum amount of data that can be sent out on a connection without being acknowledged. HSTCP is the very important protocol that will be used only when congestion window increases abruptly. To improve the retransmission time and to avoid congestion in completely dynamic conditions where node mobility is completely random is the main concern of our research work.
\end{abstract}

\section{Keywords}

TCP, HSTCP, Congestion, MANETs

\section{INTRODUCTION}

Ad hoc networks are the infrastructure less networks that are easily deployable and self configuring, with each constituent node behaving like a transceiver. Ad hoc wireless networks, due to their quick and economically less expensive deployment, which has applications in several areas. Mobile Ad Hoc Network (MANET) is wireless connectivity through the nodes constructed by the actions of the group of nodes, which normally has a dynamic shape and limited resources [1].

MANET is more appropriate in the present day scenario as the field of mobile communication is rapidly growing. The fact that MANET is distributed, makes it more favorable for the ever need to have continuous communication. It also addresses the major problem of shortage of frequencies due to blooming carriers which are a major overhead. The other noteworthy point is that the nodes are not under the impersonation of any network carrier or under the influence of a monitored carrier.

However due to the above mentioned characteristics there arises challenge for implementation of the existing traditional connecting protocols. The fact that Ad-hoc networks are connectionless and distributed opens up scope

for creation of new protocols that needs to take care of the emerging problems such as

1) Finding the nearest /neighboring nodes in network

2) Energy constraints of the nodes

3) Path discovery b/w source and destination

4) Congestion due to frequent path breaks

\section{5) Node mobility}

The above factors are dealt with in detail in [2][3]. Now moving on to mobility and non mobility of the wireless nodes, both the terms can be explained in military application. For instance-the sensor nodes which may be placed at the borders for intrusion detection may be non mobile at particular critical point. This serves as an example for mobile node which are made static due to particular scenario. Moving on, the soldiers in the battle fields may use Ad-hoc nodes to form connectionless and dynamically self configuring connection in order to communicate among the various stations while on the go. The most important aspect to be handled is congestion in case of MANET. Main reason for congestion is more number of packets that the network can handle. So, the motto of congestion control is to maintain the number of packets in the network. Congestion in Ad-hoc network can happen due to various reasons like (i) limited bandwidth (ii) due to mobility (iii) algorithm used for routing (iv)node failure. The protocols employed in MANET should cope up with all the above reason for congestion separately or in unison [5]. The paper deals with the concepts of congestion and optimization techniques implemented in modern day protocols to handle congestion.

TCP and UDP are the most important protocols of the transport layer, to establish reliable connection between the sender and receiver. However among the two, TCP is more reliable as we have an acknowledgement which is a proof that the receiver has received the data, which is not present in the later one. The protocols of transport layer are not 100\% accurate but they tend to provide "Best effort Service". TCP uses a 3 way handshake mechanism to establish connection between the source and destination. There are a lot of variants in TCP like TCP Reno, TCP New Reno, Vegas, Westwood, CUBIC, Scalable, FAST, HSTCP etc.

In the paper mainly concentrate on HSTCP usage in MANET.

\subsection{HSTCP}

High speed TCP was proposed in 2003 to improve the performance of TCP connections with large congestion windows. It introduces a relation between an average congestion window and packet drop rate [7]. If packet drop rates are more than $10^{-3}$ it follows the traditional AIMD algorithm by increasing congestion window $(\mathrm{CW})$ as in (1) and by decreasing $\mathrm{CW}$ as in (2).

$$
\begin{aligned}
& C W=C W+1 / c w n d \ldots \ldots \ldots \ldots \ldots \ldots \ldots \ldots \ldots \text { (i) } \\
& C W=\text { cwnd } \ldots \ldots \ldots \ldots \ldots \ldots \ldots \ldots \ldots \ldots \ldots \ldots \text { (ii) }
\end{aligned}
$$

If in case, the packet drop rate becomes lesser than $10-^{3}$, HSTCP adopts a more aggressive increase/decrease algorithm.

Routing is a mechanism of finding the optimal path to communicate with the source and destination. It plays a vital 
role in Ad-hoc networks due to the fact that they are distributed. The efficient working of the protocol depends on the path chosen to reach to the destination, considering the mobility of the nodes. The routing protocols under our scope are with respect to routing info update mechanism. Once again they are further classified into
(i)
Proactive [Table drive]
(ii)
(ii) Reactive[On demand] (iii) Hybrid

In case of proactive protocols, there is a usage of routing table. And the best path between sender and receiver is determined by the table entries. For example: DSDV, CHGSR, WRP

In case of reactive protocols, the routing is initiated by sender or receiver and each neighboring node is treated as a potential path to reach the destination. For example: DSR, ABR, TORA, AODV

\subsection{AODV}

AODV (Ad-hoc On-demand Distance Vector) routing protocol uses an on demand approach for finding routes i.e., route is established only when it is required by a source node for transmitting data packets. It makes use of destination sequence numbers to identify the most recent path. Here, the source node and the intermediate nodes store the next-hop information corresponding to each flow for data packet transmission. The source node floods the RouteRequest (RREQ) packet in the network when a route is unavailable for the required destination. It may obtain multiple routes to different destinations from a single RouteRequest. All intermediate nodes having valid routes to the destination, or to the destination node itself, are allowed to send RouteReply (RREP) packets to the source. Every intermediate node, while sending a RouteRequest, enters the previous node address. This is the brief working of AODV protocol.

\section{RELATED WORK}

TCP is the most trusted protocol in wired network and has been widely adopted as a reliable transfer protocol for most of the existing communication networks. However the same doesn't implies for Ad-hoc networks as its infrastructure less and self configuring with mobile nodes. The basic idea of end to end connection fails and continuously established links for data transfer are not available due to wireless transmission. TCP needs modifications for the existing protocol and help of routing protocols in order to adapt for the MANET.

Most of the Ad-hoc networks use TCP variants in transport layer for connection. In this paper mainly highlighting the usage HSTCP.

TCP Reno is designed in such a way that when a congestion occurs in the network the Congestion Window decrease to half of its initial value equation (ii). Else the window size increases exponentially as pointed out in equation (i). The working principle of this protocol is pretty straight and is in accordance with the AIMD. Assume that the packet loss rate is $p$, the current Standard TCP's average congestion window is roughly

$$
\text { Avg. } \mathrm{CW}=1.2 / \sqrt{\mathrm{p}}
$$

Segments in accordance with the standards. This forms a serious limiting factor on the congestion windows that can be achieved by TCP in realistic usage [5]. For example(1), for a Standard TCP connection with 1500byte packets and a $100 \mathrm{~ms}$ RTT, achieving a steady-state throughput of $10 \mathrm{Gbps}$ would take an average congestion window of 83,000 segments, and a packet drop rate of at most one congestion every $5,000,000,000$ packets.

This scenario is widely acknowledged as an unrealistic constraint. To address this limitation of TCP, HSTCP was proposed in 2003 by S. Floyd [5].

As mentioned in the earlier introduction to HSTCP we discussed only relating case where the packet drop rate was more than 10-3 but if the packet drop rate was lesser than 10-3 .For this case, the more aggressive increase/decrease mechanism is adapted by HSTCP. When an ACK received, $\mathrm{CW}$ is updated as

$\mathrm{CW}=\mathrm{CW}+\mathrm{a} / \mathrm{cwnd}$

When the congestion is detected the window size is updated as shown below,

$\mathrm{CW}=\mathrm{CW}-\mathrm{b}(\mathrm{CW}) * \mathrm{CW}$

The canonical values for $\mathrm{a}$ and $\mathrm{b}$ constants are 1 and 0.5 respectively. As the $\mathrm{CW}$ size increases beyond fixed threshold, the value of $\mathrm{b}$ decreases from 0.5 to 0.1 and the value of ' $a$ ' increases respectively [5].Study on HSTCP is presented in detail manner in paper [6] [7].

\section{METHODOLOGY}

In the methodological design of HSTCP need to concentrate on the fact that there are other users in the network and HSTCP needs to co-exist with them. The proposed system needs to shift from TCP Reno to HSTCP when the connection is of high-speed or not. This is detected by congestion window value. As mentioned in concepts of HSTCP the probability of packet drop, if lesser than 10-3 then window size will be 38. This clearly shows that TCPReno cannot achieve CW size greater than 38 for lower bandwidth. In accordance with the equations (iv) and (v) the values of a and b needs to be chosen to get $\mathrm{CW}$ size nearly equal to 83000 to achieve 10Gbps throughput. Considering throughput of TCP when using $\mathrm{CW}=\mathrm{W}$

$\mathrm{W}$ packets $=\mathrm{W} * 1500 * 8$ bytes

Here 1500 bytes is the payload in TCP segment as referred in example (1).Throughput of the TCP considering W Throughput $=\mathrm{W} * 1500 * 8 / 0.1$

$=120,000 * \mathrm{~W}$ bits $/ \mathrm{sec}$.

In equation (vii) 0.1 is the assumed RTT.

As assumed in the example (1) the study state throughput to be achieved is 10Gbps, considering equation (vii)

$$
\begin{aligned}
& 120,000 * \mathrm{CW} \text { bits/sec }=10 \mathrm{Gbps} \\
& \rightarrow \mathrm{CW}=10 \mathrm{Gbps} / 120,000 \\
& \rightarrow \mathrm{CW} \sim=83000
\end{aligned}
$$

The HSTCP increase or decrease algorithm must allow CW to reach 83,000 with the packet drop probability of $p=10$ 7.TCP throughput and $\mathrm{CW}$ is as below:

Average TCP throughput $=\sqrt{\frac{a(2-b)}{2 b p}} * \frac{1}{\text { RTT }} \ldots .$. (viii)

Average TCPCW $=\sqrt{\frac{a(2-b)}{2 b p}}$

Considering packet loss probability equal to $10-7$ and from equations (iv) and (v), the $\mathrm{a}$ and $\mathrm{b}$ satisfy the relationship: 


$$
83,000=\sqrt{\frac{a(2-b)}{2 b} * 10^{7}}
$$

Possible solutions 1 by assuming values $a=64$ and $b=0.09$

$$
\begin{aligned}
& =\sqrt{\frac{64(2-0.09)}{2 * 0.09} * 10^{7}} \\
& =\sqrt{\frac{64 * 1.9}{0.18} * 10^{7}} \\
& =\sqrt{679111111} \\
& =82408.198 \text { (also approximately } 83000)
\end{aligned}
$$

Possible solutions 2 by assuming values $a=459$ and $b=0.5$

$$
\begin{aligned}
& =\sqrt{\frac{459(2-0.5)}{2 * 0.5} * 10^{7}} \\
& =\sqrt{\frac{459 * 1.5}{1.0} * 10^{7}} \\
& =\sqrt{688.5 * 10^{7}} \\
& =82975.90(\text { approximately 83000) }
\end{aligned}
$$

Choosing appropriate values for $\mathrm{a}$ and $\mathrm{b}$ by above possible solutions 1 and 2 , considering the $\mathrm{CW}$ update method by solution 1:

- If there are no packet loss and RTT $=0.1 \mathrm{sec}$, the $\mathrm{CW}$ will increase to 64 packets

- If there are packet losses in a round of transmission, the $\mathrm{CW}$ will decrease by $9 \%$ i.e, $0.09 * \mathrm{CW}$.

Considering the $\mathrm{CW}$ update method by solution 2:

- If there are no packet loss and RTT $=0.1 \mathrm{sec}$, the CW will increase to 459 packets

- If there are packet losses in a round of transmission, the $\mathrm{CW}$ will decrease by $50 \%$ i.e, $0.5^{*} \mathrm{CW}$.

Considering the above factors the designer of HSTCP [5] has chosen the solution 1 values of $a$ and $b$ i.e. $a=64$ and $b=$ 0.09 as the most suitable values.

\section{RESULT ANALYSIS}

Here we are comparing the QoS parameter, of the congestion window plotted versus the time. We compare the change in the congestion window by getting the present time and congestion window size by following code snippet

Set cwnd [\$tcpSource set cwnd_]

puts \$file "\$now \$cwnd"

\$ns at [expr \$now+\$time] "plotWindow \$tcp Source \$file"

\$ns at 10.1 "plotWindow \$tcp \$windowVsTime2"

The above code writes the present time at interval of 10.1 and the congestion window size at that particular duration in to the window file. The plot is made for two conditions (i) for wired network (ii)for Ad hoc network. The Figure 4.1 and Figure 4.2 show the plotted values using xgraph tool.

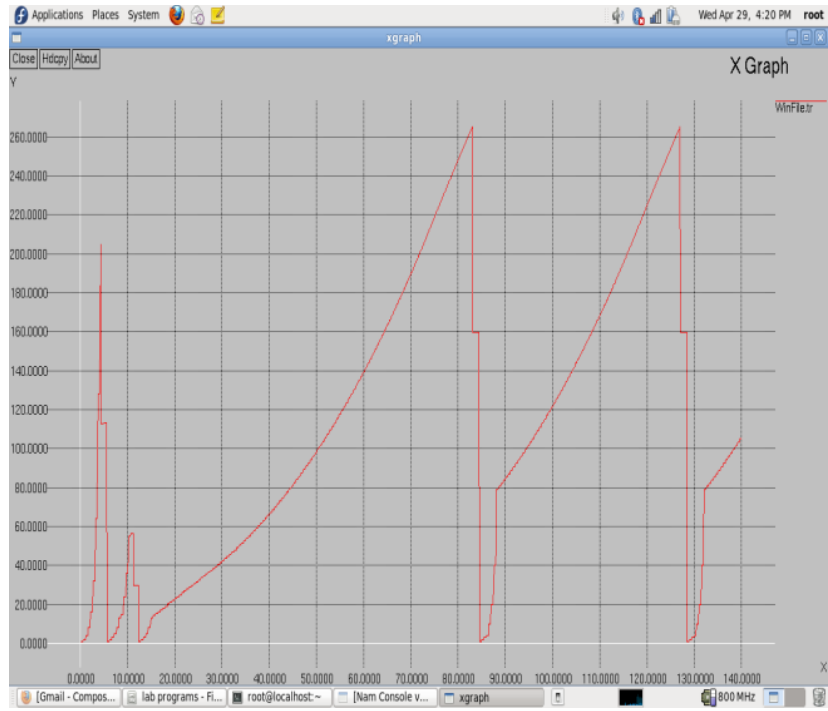

Fig 4.1 HSTCP for Wired Networks

The Fig4.1 shows the changes in the window size throughout the transmission. We can see some consistency in the plot because of the wired connection that exists between source node n0 and destination node $\mathrm{n} 4$. The time period of execution is same for both the procedures and is set to 500 .

We observed in these cases changes entirely when it comes toAd hoc networks because of the various factors discussed in the introduction. For the implementation in case of wireless networks we are considering AODV protocol and the plot of congestion window is divided as (1) Ad hoc networks without mobility (2) Ad hoc networks with random mobility

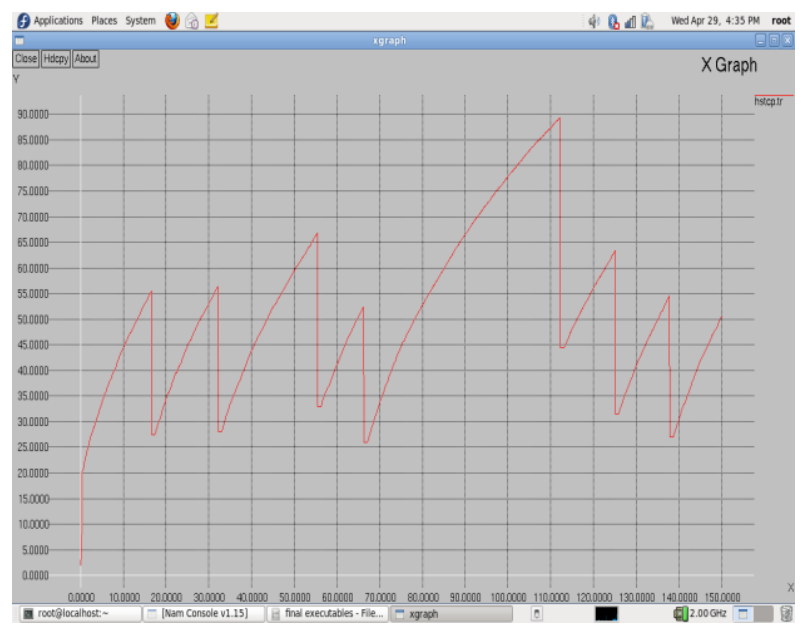

Fig 4.2 HSTCP for Ad-hoc Networks

The Fig 4.2 shows the congestion window changes applied in Ad hoc networks. Here the nodes are not mobile but both the protocols are applied. The congestion window drop rate is less in case of HSTCP when compared to Reno. The cwnd is not as consistent as in wired network because of wireless mode of transmission.

We have reached the max limit of 80000 as from the use of $\mathrm{cw}$ increase factors $\mathrm{a}(\mathrm{w})$ and decrease factor $\mathrm{b}(\mathrm{w})$. The values changes in the HSTCP enable it to reach to the theoretical value approximately of about 83000 . The inconsistencies in the figure are due to frequent path breaks in Ad hoc network, due to mobility of nodes. The factor to be considered is the 
increase in the cw size which is rapid in case of HSTCP when compared with that of the traditional TCP approach applied.

The analysis is clear proves that the window size changes dynamically and more sharply in case of HSTCP rather than in traditional methods and thereby leading to a larger window size for the large data transfer when applied in Ad hoc networks

\section{CONCLUSION}

From the result analysis the test cases its instantiated that the HSTCP when used along with the changed ' $a$ ' and ' $b$ ' parameters(a-increase parameter $b$-decrease parameter) produces a better congestion window increase rate and also tends to recover faster from a congestion. As the working environment is Ad-hoc we are bound to encounter frequent path broken due to node mobility. So its pretty much essential to have faster recovery from the congestion. From the result analysis it's evident that the better window size is achieved over shorter period. Even if the nodes are static there exists the problem of transmission range and selecting the optimal path to reach the destination. The maximum congestion window size of 83,000 as calculated for the respective 'a' and ' $b$ ' values of 64 and 0.09 is approximately attained. Fact that the computed values when applied in actual simulation are accordance. Hence HSTCP outperforms compared with Reno both in wired and wireless networks.

In our further research work, we will consider various network parameters for different TCP congestion control variants and to bring with an efficient congestion control technique for ad hoc networks there by which increases the performance of the network.

\section{REFERENCES}

[1] Saleh Ali K.Al-Omari , Putra Sumari An overview of mobile Ad hoc networks for the existing protocols and applications, International journal on application of graph theory in wireless ad hoc networks and sensor networks(graph-hoc),Vol.2,No.1,March

[2] Ahmad Al Hanbali, Eitan Altman, Philippe Nain, A Survey of TCP over Ad Hoc Networks, INRIA, 2011Tavel, P. 2007 Modeling and Simulation Design. AK Peters Ltd.

[3] Imrich Chlamtac a , Marco Conti b, Jennifer J.-N. Liu "Mobile ad hoc networking: imperatives and challenges ElsevierA d Hoc Networks 1 (2003) 13-64

[4] S Floyd, Highspeed TCP for large congestion windows, RFC 3649 experimental, 2003Brown, L. D., Hua, H., and Gao, C. 2003. A widget framework for augmented interaction in SCAPE.

[5] G. Holland and N. Vaidya, Analysis of TCP Performance over Mobile Ad Hoc Networks, ACM/IEEE MOBICOM '99, Seattle, Washington, Aug. 1999.Spector,

[6] Gururaj H L, Ramesh B, An efficient Switching TCP(STCP) Approach to avoid congestion in d-hoc networks), at IACC-2015, Bangalore, published in IEEEXplore Digital Library ISBN: 978-1-4799-8047-5, June 2015.

[7] Subramanya P, Vinayaka K S, Gururaj H L, Ramesh B

Performance Evaluation of High Speed TCP Variants in Dumbbell Network IOSR (IOSR-JCE)e-ISSN: 2278 0661, p-ISSN: 2278-8727Volume 16, Issue 2, Ver. VI (Mar-Apr. 2014), PP49-53. 